\title{
La Voix, la radio et le sermon. Censure et résistance dans The Dew Breaker d'Edwidge Danticat
}

The Voice, the Radio and the Sermon. Censorship and Resistance in Edwidge Danticat's The Dew Breaker

Florence Labaune-Demeule

\section{OpenEdition}

\section{Journals}

Édition électronique

URL : http://journals.openedition.org/transtexts/1401

DOI : $10.4000 /$ transtexts. 1401

ISSN : 2105-2549

Éditeur

Gregory B. Lee

Référence électronique

Florence Labaune-Demeule, "La Voix, la radio et le sermon. Censure et résistance dans The Dew Breaker d'Edwidge Danticat », Transtext(e)s Transcultures 跨文本跨文化 [En ligne], 15 | 2020, mis en ligne le 14 décembre 2020, consulté le 27 janvier 2021. URL : http://journals.openedition.org/ transtexts/1401; DOI : https://doi.org/10.4000/transtexts.1401

Ce document a été généré automatiquement le 27 janvier 2021.

(c) Tous droits réservés 


\section{La Voix, la radio et le sermon. Censure et résistance dans The Dew Breaker d'Edwidge Danticat}

The Voice, the Radio and the Sermon. Censorship and Resistance in Edwidge

Danticat's The Dew Breaker

Florence Labaune-Demeule

1 Dans l'article intitulé «Censorship and Literature » qui constitue une entrée de l'oxford Research Encyclopedia of Literature, Nicole Moore écrit :

Contemporary scholarship emphasizes the dynamic interplay between literary expression and forms of cultural regulation, recognizing its paradoxically productive capacity to generate as well as suppress meaning. At the same time, accounting for censorship's role in the history of the world's literature means coming to grips with the often brutal repression, prohibition, and persecution of writing, writers, performance, and cultural producers by sovereign power underwritten by violence. ${ }^{1}$

2 Les liens entre censure et littérature sont donc des liens intrinsèques, qui ont pour vocation de produire ou de soustraire du sens, de livrer ou de taire la signification du monde, et ce par de multiples biais, dans un contexte de pouvoir fort, reposant souvent sur la violence.

De nombreux auteurs postcoloniaux ont été directement ou indirectement soumis à certaines formes de censure, et pour certains fort récemment, quels que soient les contextes et les types de censure envisagés - censure politique, religieuse, linguistique et culturelle, mais aussi censure insidieuse, pour n'en mentionner que certaines. Pour les écrivains postcoloniaux, dont le but a souvent été de proposer un contre-discours, braver la censure constitue toujours un risque, voire un danger, et Martin Munro montre bien que les parents d'Edwidge Danticat craignaient, pour cette raison, de la voir devenir écrivain. ${ }^{2}$ Personne n'aura oublié la fatwa décrétée contre Salman Rushdie par l'Ayatollah Khomeiny en 1989 après la publication des Versets sataniques (1988), dont Rushdie lui-même a reconnu, lors des Assises Internationales du Roman à Lyon en 
2017, qu'elle est assez inexplicable : d'abord autorisé, le livre a ensuite été diabolisé, obligeant son auteur, traqué, à se cacher et à changer d'identité pour échapper au danger. ${ }^{3}$ Mais bien d'autres écrivains postcoloniaux ont dû faire face à cette censure protéiforme et leur contre-discours est bien sûr le moyen de se ressaisir d'une parole rendue impossible. ${ }^{4}$

Edwige Danticat, quant à elle, prend pour objet de récit la dictature des Duvalier père et fils sévissant à Haïti, de 1957 à 1971 pour François Duvalier dit « Papa Doc », et de 1971 à 1986 pour son fils Jean-Claude, «Baby Doc ", une ère d'atrocité que les parents de l'auteure ont fuie.

Dans le roman publié en 2004, The Dew Breaker, Danticat situe l'action des neuf chapitres à Haïti ou aux États-Unis, dans les communautés haïtiennes de New York ou de Floride, pendant la dictature Duvalier et pendant les années suivantes. ${ }^{5}$ Le titre de l'ouvrage est une référence explicite aux Tontons Macoutes, autre nom des Miliciens, les Volontaires pour la Sécurité Nationale, hommes forts du régime.$^{6}$ Les chapitres du roman, qui ont pour la plupart également été publiés sous forme de nouvelles avant d'être ainsi réunis dans un roman, sont thématiquement liés par la figure du briseur de rosée et celle des membres de sa famille - son épouse, Anne, et sa fille $\mathrm{Ka}-$, puisque trois chapitres présentent chacun un pan de l'histoire du tortionnaire, contée à rebours de la chronologie, de la confession qu'il est contraint de faire à sa fille plusieurs décennies plus tard (dans le premier chapitre, intitulé "The Book of the Dead»), au poids du secret si lourd à porter pour sa femme, Anne, qui se réfugie dans la foi dans le chapitre quatre, "The Book of Miracles ", pour revenir ensuite aux premiers épisodes tragiques rapportés quant à eux dans le chapitre final éponyme, "The Dew Breaker ». On y apprend que le père, Tonton Macoute, a torturé et mis à mort le demi-frère d'Anne, un pasteur, avant de faire croire à son suicide. Avant de mourir, le pasteur avait défiguré le briseur de rosée, et Anne, venue s'enquérir du sort de son demi-frère, prend le tortionnaire pour une victime, le soigne puis l'épouse.

Les trois nouvelles directement consacrées à l'histoire du briseur de rosée sont donc présentées dans un rebours chronologique qui met en valeur la censure à l'œuvre : si le lecteur perçoit d'emblée les ressassements, les scrupules, la honte et la culpabilité du père dans la première nouvelle, il en vient bientôt à se focaliser sur la difficulté de l'aveu. Entre détours et circonvolutions, litotes et métaphores, la vérité finit par poindre. Le père s'autocensure pour ne pas avouer ses crimes, et censure toute expression - même artistique - de sa fille, qui pourrait s'avérer dangereuse pour lui. Cette dissimulation est l'enjeu de la seconde nouvelle : le non-dit fait de la famille une cellule où la parole est ambiguë, entre révélation et silence, le discours étant strictement contrôlé. Au contraire, la troisième nouvelle consacre la voix et l'oralité, tant pour les personnages en jeu que pour le lecteur, qui parvient à accéder à la vérité des faits dans leur enchaînement causal. L'autocensure n'est plus de mise et seule domine la censure du pouvoir politique qui proclame l'interdiction de dire ce qui n'est pas conforme, ce contre quoi s'élève le contre-discours du pasteur dissident.

7 Le lecteur peut ainsi retisser les liens qui structurent et suturent un roman dont Mary Gallagher écrit que le trait distinctif est «sa poétique de fragmentation », construite sur une apparente discontinuité narrative et thématique :

While the principal instinctiveness of Edwidge Danticat's third novel lies undoubtedly in its poetics of fragmentation [...] it would be a distorsion to suggest that this book is innovative on a formal level only. [...] This structure raises many questions, but rather than asking how successful the book is as a novel, and to what 
extent it remains a collection of stand-alone units that create a sense more of discontinuity than of consequential development of character and plot, readers may find themselves searching for secret passages connecting the eponymous plot and the individual stories, even if the author might not seem to have provided enough keys to identify the links. This hermeneutic shortfall opens up the meaning of the individual stories, the suspicion of a hidden continuity lending each story additional, if uncertain, depth. Moreover, the fragmentation of the book's structure itself enacts the brokenness of the lives portrayed and performs the concealment, displacement, and disconnection that the book also figures thematically. ${ }^{7}$

Dans un premier temps, on questionnera le lien entre la voix et la censure, ce qui conduira, dans un second temps à montrer comment le trauma lié à la censure et à la violence dictatoriales rend la parole impossible, condamnant victimes et bourreau à une autocensure et à des formes indirectes de discours. Enfin, on s'interrogera sur la narration comme discours de révélation reposant paradoxalement sur des stratégies de discours implicite.

\section{La Voix, la radio et le sermon : discours autoritaire et contre-discours}

S'inscrivant dans la perspective de la tradition caribéenne qui accorde une place prépondérante à l'oralité, l'auteur accorde ici à la voix une importance à la fois thématique et structurelle inhérente à la nouvelle finale, où la voix doit être analysée en regard de la dissimulation de la voix dans les autres nouvelles.

Ici, l'auteur établit un lien entre censure et oralité, montrant que l'expression d'une idéologie totalitaire passe par la voix mais que c'est aussi par cette dernière que s'exprime une contre-idéologie. Au discours dominant du dictateur fait ainsi écho le contre-discours de la dissidence que représente le pasteur dans cette nouvelle, dernière du roman rappelons-le, mais première dans la chronologie des faits.

11 Lorsque le futur tortionnaire, âgé de dix-neuf ans, avait été réquisitionné dans sa campagne pour venir écouter un discours du dictateur à la capitale, c'était la voix du président, aux accents nasillards, qui avait joué le rôle d'élément hypnotique à l'origine de la conversion du jeune homme et de son enrôlement chez les Volontaires. La voix du dictateur apparaît tout d'abord comme désincarnée et les auditeurs forcés, au nombre desquels figure le jeune homme, sont eux aussi dépersonnalisés : ils ne sont que des corps, dont la présence en masse a pour simple but d'impressionner. ${ }^{8}$ Assis en plein soleil pendant des heures à écouter un discours interminable, le jeune homme parvient à mémoriser quelques arguments seulement, sanguinaires et simplistes, comme le montre la structure emphatique et répétitive du discours :

If anyone tried to topple him, the president threatened, blood would flood in Haiti as never before. The land would burn from north to south, east to west. There would be no sunrise and no sunset, just one big flame licking the sky. ${ }^{9}$

L'expérience épiphanique, presque mystique, que connaît ensuite le jeune homme montre le pouvoir hypnotique, envoûtant, de la voix présidentielle :

After the third, fourth, or fifth hour of the speech, he found himself dreaming. He thought he saw a flock of winged women circling above the palace dome, angry sibyls ranging in hue from cinnamon, honey, bronze, sable, to jet-black, hissing through the rest of the speech. 
Later he would [say], 'I thought they were angels, caryatids, maybe a soul for each of us standing there in the sun. ${ }^{10}$ One ", «the Renovator of the Fatherland " utilise, comme moyen d'expression de prédilection, la radio, organe de diffusion directe et massive du discours de terreur et de sa violence, comme lorsque est annoncée l'exécution de dix-neuf jeunes officiers :11

The president [...] had been heard on the radio announcing the execution of nineteen young officers, members of the palace guard, whom the president thought had betrayed him. The president [...] had listed the officers' names, roll-call style, on the radio, had answered 'absent' for each of them, then had calmly announced, 'They have been shot.' ${ }^{12}$

Pour le briseur de rosée, tout personnage d'autorité représente une figure parentale, que ce soit la figure paternelle du président, quasi divine dans son omnipotence, ou celle de Rosalie, figure maternelle qui lui chante la comptine " Fillette Lalo », décrivant une dévoreuse d'enfants qui enlève ces derniers pour les mettre dans son havresac et qui n'est autre qu'un prolongement féminin du mythique personnage de Tonton Macoute, lui-même représentant une autre vision du père de la nation. ${ }^{13}$ Si la forme passive « he was told» laisse entendre d'abord une autorité indéterminée, il apparaitt bientôt que les ordres sont ceux de Rosalie, qui copie la prononciation nasillarde du président et exprime ses directives sans ambages, en discours direct, avec des impératifs ou des propositions interrogatives, sur le mode caractéristique du parent grondant un enfant, où le pronom de deuxième personne "you " prend un sens accusateur et distancié : ${ }^{14}$

'By all accounts, the arrest turned into a cockfight,' she said. [...] 'You went into a church filled with people when you could have gotten him on the street. Why did you bring him [the preacher] here?' [...]

'You wanted him to suffer,' Rosalie was saying, smirking almost as if in admiration. 'You took too many liberties. You disobeyed.' [...]

'He's your responsibility,' she told him, turning on her heel, as if for a military-style about-face. 'I've seen him and he looks very bad. Under no circumstances should he die here. ${ }^{15}$

Les verbes de procès dynamique montrent le rôle actif joué par le briseur de rosée et soulignent sa responsabilité.

Sous la dictature, les termes du quotidien eux-mêmes prennent une valeur bien différente. Il n'est pas anodin que le tortionnaire appelle ses interrogatoires violents des « interviews ", comme pour montrer que le discours ne peut plus conduire qu'à une normalisation de la violence : le choix de l'euphémisme permet de voiler la violence tout en l'exposant.

Par le discours politique exprimé lors de meetings forcés, par la diffusion en direct des exactions du régime à la radio, par la bouche des hommes ou femmes de main du pouvoir, le discours direct semble l'apanage des puissants. Ainsi, lorsque le prédicateur emprisonné tente de répondre à l'injonction d'une voix inconnue ("'Hey, preacherman!' a voice was calling from outside the dark cell. 'Come on over here!'»), il reconnaît immédiatement cette voix comme la personnification de l'autorité politique, la voix devenant bientôt "The Voice", personnage sans corps identifiable mais inspirant la peur, incarnation d'un monstre bestial, comme le laisse supposer le pronom it qui y réfère. ${ }^{16}$ De nouveau, c'est le discours direct qui la caractérise, avec ses apostrophes, ses interjections et ses impératifs : 
'Hey, preacherman!' a voice was calling from outside the dark cell. 'Come on over here!' [...]

'You,' the Voice called out to the others inside the cell. 'Bring the new prisoner here.' [...]

'You're a lucky man,' it was saying. 'This is your lucky day, you lucky man.' [...]

'Hurry up or I'll leave you here,' the Voice said. ${ }^{17}$

Or, cette bestialité s'insère aussi dans le discours religieux du pasteur dissident et l'on observe que les moyens d'expression de son contre-discours reprennent exactement les mêmes conventions, avec quelques particularités. C'est en effet lors de prêches à la radio ou lors de sermons dans son église - tous deux des types de discours publics qu'il dénonce la brutalité du régime en l'associant au Diable, à la Bête de l'Apocalypse. Le religieux dissident présente en effet une émission tous les dimanches sur une radio au nom allégorique, Radio Lumière, où l'appel à combattre le tyran est à peine voilé : il y fait par exemple l'éloge d'opposants politiques du passé ou utilise des épisodes bibliques comme paraboles, soulignant ainsi la capacité des plus humbles à lutter contre les plus puissants. ${ }^{18}$ Après un résumé en style narrativisé, c'est de nouveau le discours direct qui est utilisé pour montrer la force de persuasion du pasteur et l'impact que ses mots peuvent avoir, représentant en cela une menace pour le régime du président :

He exalted Queen Esther, who had intervened to halt a massacre of her people; Daniel, who had tamed lions intended to devour him; David, who had pebbled Goliath's defeat; and Jonah, who had risen out of the belly of a sea beast.

'And what will we do with our beast?' the preacher encouraged his followers to chant from beside their radios at home, as well as from the plain wooden pews of his sanctuary.

He liked to imagine the whole country screaming, 'What will we do with our beast?' but instead it seemed as if everyone was walking around whispering the sanctioned national prayer, written by the president himself: 'Our father who art in the national palace, hallowed be thy name. Thy will be done, in the capital, as it is in the provinces. Give us this day our new Haiti, and forgive us our anti-patriotic thoughts, but do not forgive those anti-patriots who spit on our country and trespass against it. Let them succumb to the weight of their own venom. And deliver them not from evil. ${ }^{19}$

19 La joute verbale entre représentants du pouvoir et dissidents semble pourtant bien inégale: au pronom collectif inclusif we du pasteur répond la nouvelle prière du pouvoir, où certes domine le pronom de deuxième personne à valeur biblique thou. Dans ce Pater Noster inversé, le président, nouvelle figure divine, reçoit les suppliques de ceux qui, lui rendant hommage, s'auto-désignent comme ses subalternes. Dans le même temps, les opposants au pouvoir sont décrits comme les représentants des forces du mal, comme le suggère l'image du venin, donc du serpent, faisant d'eux des damnés. Le pasteur est conscient des risques :

Ever since he'd begun broadcasting his radio show and had lost his wife, perhaps as a result of what he said on the air, the preacher had grown accustomed to these displays of fearful affection and had hence learned that the best way to appease them was to maintain his calm, while citing Bible passages, almost as incantations to soothe those who thought they could save his life. [...] He'd dreamed his own death so many times that he was no longer afraid of it. ${ }^{20}$

La narration oppose ici le discours direct, lié à l'homme public et au pouvoir, et le discours indirect, réservé aux pensées intimes. Ainsi, lorsque le pasteur partage ce qui sera son dernier repas avec ses diacres, les propos qu'il exprime en discours direct tranchent avec le discours indirect libre de ses pensées (" 'I know my God and I'm 
placing myself in His hands' »). ${ }^{21}$ Prononcées alors qu'il mange le pain de son dîner, les paroles au discours direct permettent d'établir un parallèle avec la Cène : «'It's time for the service. [...] I don't think you three should walk with me to the church tonight. I'll walk alone.' ».22 Comme lors de l'Eucharistie, la puissance performative du Verbe permet l'incarnation. Le sacrifice du prêtre est christique en ce qu'il est public, et accepté au nom de la communauté :

What [the deacons] didn't realize, or didn't want to acknowledge, was that he'd already decided to give his life, had made a pact with Heaven to be sacrificed for his country. ${ }^{23}$

Et c'est en effet en homme public, connu, reconnu et salué par toutes les petites gens de la rue, que le prêtre se rend à l'église pour la dernière fois. De nouveau, c'est en discours direct que ses propos sont rapportés et l'apostrophe « pastor » utilisée par les membres de sa congrégation alors qu'il est en chemin pour l'église montre qu'il est reconnu comme le gardien du troupeau. Mais lorsqu'il est sur le point de mourir, ses pensées sont de nouveau exprimées en discours indirect libre. ${ }^{24} \mathrm{Et}$ si les paroles rapportées en discours direct lui confèrent ce statut de martyr que le gouvernement craint tant, le doute qui finalement l'étreint et les pensées intimes, exprimés en discours indirect libre, font de lui bien plus qu'un martyr - un homme martyrisé, ce qui accroît l'empathie du lecteur.

On observe donc que le discours public, qu'il soit propagande en faveur du régime en place ou qu'il soit dissidence, s'exprime par des moyens et des biais identiques. La radio est le moyen d'expression de masse qui sert à imposer la censure ou à la combattre. En revanche, pour le pasteur comme pour le briseur de rosée, les discours privés, les pensées sont exprimés essentiellement en discours indirect libre, ce qui permet de mettre en exergue la complexité de leurs personnalités et la dualité de chacun tout en en rendant la source opaque, les protégeant potentiellement de la répression. C'est donc a priori par des moyens identiques que Danticat exprime tout à la fois un point de vue et son contraire, de sorte que chacun est perçu comme un écho de l'autre, une réverbération en miroir, dont on verra qu'elle caractérise toute la poétique de Danticat dans ce roman.

Or, si les deux personnalités antagonistes que sont l'homme de main d'une part, et le dissident d'autre part, peuvent se saisir de la parole publique, les autres personnages du roman sont avant tout décrits comme des êtres balbutiants, voire bègues, pour qui la communication a été rendue impossible par le trauma.

\section{Le trauma, ou l'impossible parole : censure, autocensure et faillite de la voix}

24 Les conséquences du trauma se manifestent en effet par une autocensure qui peut s'avérer volontaire ou involontaire, c'est-à-dire que l'autocensure peut se faire mensonge, dissimulation consciente ou reflet d'une communication rendue impossible par le trauma.

Dans le premier cas, l'autocensure est consciente et les personnages concernés savent que d'elle dépend leur survie. C'est bien sûr le cas du briseur de rosée et de son épouse Anne qui doivent, pour survivre et échapper à la vengeance, s'exiler à New York et censurer leur discours et leurs gestes afin de préserver leur identité nouvelle et de 
rendre indétectables leurs fautes passées. Notons incidemment que cet ancien briseur de rosée se fait appeler, dans la première nouvelle, M. Bienaimé, nouvelle identité masque qui le rend débonnaire. La situation d'Emmanuel Constant, dénoncée dans la nouvelle « The Book of Miracles ", est un reflet de ce qui pourrait alors les attendre : la communauté haïtienne a placardé la photo de cet autre tortionnaire dans la rue afin qu'il puisse être identifié et dénoncé pour ses crimes contre l'humanité. ${ }^{25} \mathrm{Et}$ c'est, ironiquement, la fille du briseur de rosée Ka, qui semble le reconnaitre lors de la messe de Noël à Brooklyn et veut le dénoncer. C'est aussi son père qui arrête son bras lorsque Ka cherche à interpeller l'homme. ${ }^{26}$

La vie d'Anne et du briseur de rosée est donc une vie passée en marge de leur propre communauté, sans amis ni réelles connaissances, une vie de fantômes. Ils cherchent à effacer les traces de leur présence : ainsi, pour Noël, ils refusent d'illuminer leur maison et fuient le regard des autres, comme lorsque l'affiche de Constant devant leurs fenêtres semble les dénoncer :

Still, Anne and her husband had put up no decorations, fearing, irrationally perhaps, that lit ornaments and trimmings would bring too much attention to them. Instead it was their lack of participation that made them stand out, but by then they had already settled into their routine and couldn't bring themselves to change it. [...]

Still, every morning and evening as her eyes wandered to the flyer on the lamppost in front of her beauty salon and her husband's barber shop, Anne had to fight a strong desire to pull it down, not out of sympathy for Constant but out of a fear that even though her husband's prison 'work' and Constant's offenses were separated by thirty-plus years, she might arrive at her store one morning to find her husband's likeness on the lamppost rather than Constant's. ${ }^{27}$

Pour Anne, qui souffre aussi de la culpabilité de n'avoir pu sauver son jeune frère de la noyade et d'avoir épousé l'homme qui a tué son demi-frère le pasteur, la rédemption que symbolise le changement intérieur de son mari s'apparente à ces miracles religieux dont elle est friande :

Anne was proud of her daughter, proud of her righteous displeasure. But what if she ever found out about her own father? About the things he had done?

After the sermon, the congregation got up [...] to take Holy Communion.

'How lucky we are,' said the priest, 'that Jesus was born to give his flesh for us to take into ourselves.'

How lucky we are, Anne thought, that we're here at all, that we still have flesh. [...]

It was always like this, her life a pendulum between forgiveness and regret, but when the anger dissipated she considered it a small miracle, the same way she thought of her emergence from her occasional epileptic seizures as a kind of resurrection. ${ }^{28}$

Être fascinée par les miracles est pour elle se rappeler la précarité de sa vie et avoir l'espoir d'une rédemption. Le langage religieux devient un exutoire et le seul moyen d'expression dont elle dispose pour contourner la censure qu'elle s'impose.

Pour l'ancien tortionnaire, la balafre de sa joue requiert le mensonge permanent : il lui est impossible de dire la vérité, d'une part pour protéger sa vie, comme on l'a vu, et d'autre part parce que révéler la vérité serait perdre la fille qu'il aime tant. C'est donc un autre langage qu'il doit adopter, celui de la parole détournée, celui de la métaphore ; point de langage religieux et de miracles pour lui, mais une obsession pour le «Livre des morts » de l'Égypte ancienne, où il a puisé le nom de sa fille :

A ka is a double of the body, [...] the body's companion through life and after life. It guides the body through the kingdom of the dead. [...] 'You see, ka is like soul,' my 
father now says. 'In Haiti is what we call good angel, ti bon anj. When you were born, I look at your face, I think, here is my ka, my good angel. ${ }^{29}$ est le reflet de sa double personnalité, de son passé brutal, sadique et sanguinaire et de son présent de bon père de famille aimant. Sa fille croit que les cauchemars de ce dernier sont dus à la torture subie, non à la torture infligée. Pourtant la violence du père affleure toujours, comme lorsque, agacé par le rire et les gestes de bras de sa fille au milieu de la première nouvelle, il retient le poignet de celle-ci et lui fait mal. ${ }^{30}$ Les signes, donc, peuvent s'interpréter de manière ambiguë, partielle, voire totalement fausse, mais la double signification demeure souvent présente, comme le montre la sculpture que Ka a réalisée de son père, où elle croit exprimer la souffrance du dissident qu'elle l'imagine avoir été ; or, la cicatrice du bois qui risque de se fendre représente aussi la cicatrice de la joue du tortionnaire, lacérée par vengeance. Une fois encore, ce ne sont pas les mots qui permettent d'exprimer cette vision ambiguë, voire paradoxale, mais un moyen plus artistique et silencieux - la sculpture :

I had never tried to tell my father's story in words before now, but my first completed sculpture of him was the reason of our trip. [...] It was hardly revolutionary, rough and not too detailed, minimalist at best, but it was my favorite of all my attempted representations of my father. It was the way I had imagined him in prison. [...]

I'd used a piece of mahogany that was naturally flawed, with a few superficial cracks along what was now the back. [...] I'd made no effort to sand or polish them away, as they seemed like the wood's own scars, like the one my father had on his face. But I was also a little worried about the cracks. Would they seem amateurish and unintentional, like a mistake? ${ }^{31}$

Pour le père, la révélation du passé s'opère par de multiples métaphores : après la pesée des cœurs dans l'Égypte antique, c'est la métaphore du chasseur et de la proie qu'il utilise afin de pouvoir formuler sa confession. Le choix de la narration homodiégétique et du temps présent met en relief la confession de la faute :

Each word is now hard-won as it leaves my father's mouth balanced like those hearts on the Ancient Egyptian scales.

'Ka, I was never in prison,' he says. [...]

'I was working in the prison,' my father says. And I decide not to interrupt him again until he's done.

Stranded in the middle of this speech now, he has to go on. 'It was one of the prisoners inside the prison who cut my face in this way,' he says. [...]

'This man who cut my face,' he continues, 'I shot and killed him, like I killed many people. ${ }^{32}$

Pour Ka, cette révélation provoque un réajustement de sa propre réalité puisque, repensant au rituel de confession négative qui avait lieu en Égypte antique avant la pesée des cœurs, elle prend conscience du double sens du langage et de la dualité de son père. La langue utilisée par ce dernier est une langue inversée, dont il lui aurait fallu déchiffrer les antiphrases :

Now he was telling me I should have heard something beyond what he was reading. I should have removed the negatives.

'I am not a violent man,' he had read. 'I have made no one weep. I have never been angry without cause. I have never uttered any lies. I have never slain any men or women. I have done no evil.'33

Si la réalité n'est pas facile à entendre pour Ka, en parler semble en partie censuré : lorsqu'elle appelle sa mère, le téléphone est à la fois le moyen de tenter d'avoir des 
explications et de suspendre la conversation, d'autocensurer provisoirement la révélation de l'horreur. ${ }^{34}$

Voilà justement ce que montre chacune des autres nouvelles du roman, mettant au centre de l'intrigue des personnages affectés par une forme de trauma, dans l'incapacité d'exprimer leur souffrance. L'autocensure involontaire et bien souvent inconsciente vient alors enrayer toute forme de discours de révélation, bien que les modalités en soient chaque fois différentes. Ainsi, les trois locataires du briseur de rosée, Michel, Eric et Dany, exilés haïtiens, sont tous affectés par la difficulté de dire les choses. Eric, dans "Seven ", ne parvient pas à parler de ses conquêtes féminines à sa femme, dont il a été séparé pendant sept ans, de peur de la perdre ; et lorsque, dans la nouvelle "Water Child", il cherche à joindre l'une de ces femmes, Nadine, qui a dû avorter, ses messages téléphoniques restent lettre morte sur le répondeur. ${ }^{35}$ Dans "Monkey Tails », Michel ne peut se résoudre à accepter l'identité de son père haïtien et à en parler; c'est finalement sur une cassette qu'il enregistre cette généalogie gênante pour la transmettre à son fils à naître. Dans " Night Talkers ", Dany, que ses hésitations à dénoncer celui qu'il pense avoir reconnu comme responsable de la mort de ses parents et de la cécité de sa tante, ne peut se confier à cette dernière car Estina meurt pendant la nuit. Quant à Nadine, dans "Water Child», obsédée par l'enfant qu'elle a perdu, elle se coupe de son entourage professionnel et de ses patients laryngectomisés, en qui elle voit une manifestation de son incapacité à dire son trauma, comme elle s'éloigne graduellement de ses parents restés en Haïti. Aucun moyen de communication ne peut transmettre sa douleur. Beatrice Saint-Fort, la couturière créatrice de robes de mariée, torturée à Haïti, se sent de son côté perpétuellement persécutée, contrainte de déménager, refusant de livrer à une jeune journaliste venue l'interroger sur sa carrière, ce passé indicible. Pour les trois jeunes haïtiennes de la nouvelle "The Funeral Singer ", le trauma rend la parole tout aussi difficile. Chanteuse d'enterrement, Freda a refusé de chanter au palais présidentiel et a dû s'exiler après la disparition en mer de son père, torturé.

Par conséquent, pour tous ces personnages, se saisir du discours, communiquer de manière traditionnelle et efficace est impossible, ou à tout le moins difficile. Ces personnages peuvent alors sembler produire un discours de bégaiement, qui diffère toujours la prise de parole usuelle, comme si la réalisation verbale demeurait suspendue. Ou un discours de traverse, expression qui serait plus poétique, discours métaphorique et symbolique puisque la parole leur fait défaut dans une autocensure parfois inconsciente. Discours de traverse, en effet, puisqu'ils doivent trouver des moyens détournés pour rendre la communication efficace. Pour Dany, par exemple, le fait d'être un "pallanit ", ou "night talker ", c'est-à-dire de parler la nuit sans s'en rendre compte, est un moyen déviant de communiquer quand même avec Tante Estina avant qu'elle ne meure. ${ }^{36}$ La confession impossible de jour devient effective, quoiqu'inconsciente, la nuit. Pour Michel, enregistrer l'histoire de son père et de sa propre enfance sur une cassette permet une confession à la fois intime et transgénérationnelle puisqu'elle a pour but de rendre hommage à son ami Romain, d'accepter ses origines et de transmettre son héritage à son bébé à naître. Pour Freda, Rezia et Mariselle, l'interprétation des chants funèbres ou populaires sert d'exutoire à leur mal-être, alors que pour Béatrice, créer des robes de mariée fabuleuses remplace l'angoisse qui l'habite par la beauté et l'innocence qui lui furent volées. 
36 Le lien discursif est donc subverti par le trauma qui rend l'horreur indicible, engendrant de ce fait les diverses manifestations d'autocensure auxquelles peut remédier le déplacement symbolique, voire la fiction :

For Cathy Caruth, trauma is an experience so intensely painful that the mind is unable to process normally. She claims that if the memories of the trauma return, they are often nonverbal, and the victim may be unable to describe them with words. In this connection, Cathy Caruth maintains that fiction can 'speak' trauma and may have therapeutic potential, whereas normal, discursive language cannot. ${ }^{37}$

\section{Voix narrative et censure, entre révélation et implicite - une poétique de l'écho}

Plusieurs critiques, comme Robert Birmbaum ci-après, ont montré que la poétique d'Edwidge Danticat dans The Dew Breaker était avant tout une poétique de la révélation, du dire, de l'absence de censure, donc de dénonciation de ce que fut la dictature des Duvalier, par le témoignage sans concession :

'I wanted to raise the voice of a lot of the people that I knew growing up, and this was, for the most part,... poor people who had extraordinary dreams but also very amazing obstacles.' With [The Dew Breaker], [E.Danticat's] overarching interest continues to be giving voice and attention to the neglected and marginalized people and issues. ${ }^{38}$

Danticat formule donc ce que ses personnages ne parviennent pas à mentionner sans détours, et ce pour témoigner de l'histoire et du trauma. Une fois encore, comme l'écrit Kali Tal,

[The 1]iterature of the trauma is written from the need to tell and retell the story of the traumatic experience, to make it 'real' both to the victims and to the community. Such writing serves as validation and cathartic vehicle for the traumatized. ${ }^{39}$

Ce qui parait plus original, chez Danticat, est la manière dont ce dire peut s'exprimer dans le contexte d'autocensure où évoluent les personnages. Car il n'y a, semble-t-il, dans l'écriture de ce roman, aucun désir de censure de quelque événement que ce soit, comme en témoigne la narration que fait Danticat de l'attaque réelle de l'église de son oncle à Haïti, dont on retrouve bon nombre d'éléments dans la dernière nouvelle de The Dew Breaker. ${ }^{40}$ Ni même de censure de quelque horreur que ce soit, par exemple lorsque Danticat parle explicitement d'une scène de torture de déchoukaj où un ancien Macoute s'était vu obligé de boire de l'essence avant que ses opposants y mettent le feu. ${ }^{41}$ Cependant, l'écriture de The Dew Breaker repose dans le même temps sur une poétique qui fait écho à l'autocensure manifeste des personnages face à leur trauma, une poétique du détour que l'on pourrait qualifier de poétique de la retenue. L'auteur ne cherche pas à dissimuler, mais elle préfère recourir à un moyen d'expression presque indirect, discret, qui soit le reflet des secrets indicibles des personnages, plutôt qu'à une formulation plus explicite. Et il nous semble que cette poétique de la révélation en creux et du détour repose sur plusieurs stratégies. La première est la disjonction, ou la discontinuité, que la critique a souvent mise en avant et qui se manifeste à travers la fracture structurelle observable dans le genre hybride roman-collection de nouvelles que l'on découvre ici. Les chapitres formés d'histoires disjointes sont le reflet des 
fractures psychologiques et émotionnelles des protagonistes que les blancs narratifs représentés entre les histoires semblent symboliser. Mary Gallagher y voit une illustration de l'attention que le lecteur doit porter à chacune en particulier, ainsi qu'à l'ensemble, obligeant ce dernier à établir lui-même les connexions et transitions absentes. «Danticat's writing illustrates performatively that attentive reading is required in order to make certain connections ", écrit M. Gallagher. ${ }^{42}$ Nous avons montré ailleurs que ce récit kaléidoscopique ne peut être unifié que par la voix silencieuse du lecteur qui procède au collage cohérent et significatif des chapitres/ nouvelles par une lecture performative ${ }^{43}$ Pour que ce dernier y parvienne, Danticat fait reposer sa narration sur une poétique de résonance ("A Poetics of Resonance »), créant une esthétique de réverbération (" an aesthetic of reverberation »), qui vise à tisser des relations étroites entre les personnages, les temporalités, et les événements : ${ }^{44}$

This operates most overtly at a thematic and more broadly intertextual level between the different stories. [...] Similarly, various smaller motifs are interwoven, sometimes to opaque effect, from one story to another. [...] However, the poetics of reverberation also operates at other levels in the book. For example the notion of memory is given added cultural resonance by reference to Egyptian, Japanese, and Catholic mythology and religion. [...] The motif also highlights writing itself, or the book as record, and thereby the notion of the historical record and its link to memory. ${ }^{45}$

De même, les choix linguistiques participent de cette poétique de la réverbération, qu'il s'agisse de l'utilisation des différentes langues qui se répondent (anglais et créole français notamment), ou du choix des voix narratives. Les modes d'expression du discours et des pensées des personnages se font écho d'une histoire à l'autre, mais aussi à l'intérieur de chaque histoire et, selon un troisième degré d'enchâssement, à l'intérieur de chaque personnage, comme nous l'avons vu précédemment. Enfin, la troisième stratégie majeure, semble-t-il, dans cette poétique de la réverbération, est le rejet du manichéisme, de la simplicité de la caractérisation: la profondeur des personnages, leur « rondeur " pour reprendre les termes d'E.M. Forster dans Aspects of the Novel, évite de tomber dans le stéréotype ou la caricature. Elle rend à l'homme monstrueux une part de beauté, une part d'humanité, même si cette dernière paraît parfois bien mince. Derrière tout acte barbare transparaît la possibilité d'une rédemption, d'un pardon, comme le dit Danticat. Et c'est à la fois à la part d'ombre et à la part de lumière de chacun que se consacre l'auteur: en expliquant la causalité des événements, leur imbrication logique, elle parvient à générer une forme d'empathie en creux, y compris pour le briseur de rosée et sa femme, dont le péché par omission la condamne autant que lui : refuser le manichéisme simpliste et donner aux personnages une complexité (psycho)logique, un visage double, les rend humains dans leur horreur, donc rachetables. En montrant l'horreur et son contrepoint la rédemption, en creux, par la juxtaposition des histoires disjointes mais reliées au fil de la chronologie inversée, la sympathie du lecteur reste acquise partiellement à ces personnages. Seul l'art peut autoriser cela, servir d'exutoire à la douleur et au trauma. Et dans le processus de lecture, l'auteur comme le lecteur trouvent une sorte de catharsis. Comme E. Danticat l'explique clairement, l'acte d'écriture est un acte de révélation, de mise à nu de la souffrance, et dans le même temps, il permet de supporter la réalité de l'horreur :

Jamaica Kincaid has said that if she didn't write, she'd be burning down buildings. My work allows me to exorcise my ghosts. I purge the pain from it. The words are my tears on the page. Perhaps I'd be less serene without them. I tend to be 
melancholy. The work helps me put that on the page. I lost my father and my uncle recently. The only way to grieve them was to write about them. ${ }^{46}$

Les multiples stratégies de cette poétique de la réverbération, dans le cadre du discours narratif mais aussi hors de ce dernier, fonctionnent, pour le lecteur et l'auteur, par la mise en relation de la voix narrative de l'écrit et des voix individuelles qui montent faiblement, dans leurs balbutiements hésitants, des chapitres du roman. Il s'agit là d'une poétique oxymoronique visant à traduire les cris silencieux de voix défaillantes, le silence qui résonne et la censure qui cède le pas à la liberté de parole.

Pour conclure, la narration de Danticat transcrit la parole toute puissante, celle qui censure, mais elle rend en même temps audible la parole impossible et les cris silencieux des personnages. Nous pourrions dire avec Édouard Glissant que dans The Dew Breaker,

[d]u cri fixe d'ici, déroule une parole aride, difficile [...] (Quitter le cri, forger la parole. Ce n'est pas renoncer à l'imaginaire ni aux puissances souterraines, c'est armer une durée nouvelle, ancrée aux émergences des peuples. ${ }^{47}$

4 Et d'ajouter dans le Traité du Tout-Monde, comme pour faire écho aux préoccupations de Danticat :

Écrire c'est vraiment dire : s'épandre au monde sans se disperser ni s'y diluer, et sans craindre d'y exercer ces pouvoirs de l'oralité qui conviennent tant à la diversité de toutes choses, la répétition, le ressassement, la parole circulaire, le cri en spirale, les cassures de la voix. ${ }^{48}$

\section{NOTES}

1. Nicole Moore, «Censorship and Literature ", Oxford Research Encyclopedia of Literature, Oxford University Press USA, 2016, p. 1, <literature.oxfordre.com>, accessed 12 April 2018.

2. Martin Munro (Ed.), Edwidge Danticat, A Reader's Guide, Charlottesville \& London, University of Virginia Press, 2010.

3. Salman Rushdie, Joseph Anton, A Memoir, New York, Random House Publishing, 2012.

4. Voir l'article cité plus haut de Nicole Moore, qui mentionne les noms de nombreux écrivains victimes de censure (Moore, «Censorship and Literature », p. 17).

Voir Richard Terdiman, Discourse/Counter-Discourse, The Theory and Practice of Symbolic Resistance in Nineteenth-Century France, Ithaca \& London, Cornell University Press, 1985. Voir également les questions soulevées par G. Chakravorty Spivak, Benita Parry, Sara Suleri, et bien d'autres, qui ont insisté sur l'impossible parole des subalternes.

5. Edwidge Danticat, The Dew Breaker, London, Abacus Books, 2004.

6. Le corps des Volontaires pour la Sécurité Nationale a été créé en 1958 par F. Duvalier pour assurer la protection du président. Responsables de nombreuses exactions, les Tontons Macoutes (autre nom des Volontaires) ont fait régner la terreur pendant la dictature des Duvalier. Le nom « macoute » renvoie à un personnage du folklore, représenté avec un grand sac, sorte de croquemitaine créole ; les macoutes, aussi appelés « briseurs de rosée » en raison de leurs interventions au petit jour, étaient responsables d'enlèvements, d'arrestations et de disparitions. Voir la légende de Tonton Macoute et de Fillette Lalo dans The Dew Breaker, p. 184. 
7. Mary Gallagher, « Concealment, Displacement and Disconnection. Danticat's The Dew Breaker », in Munro, Edwidge Danticat, pp. 147-160. Citation extraite des pages p. 147-148.

8. «It began when the Volunteers came to his town bussing people to a presidential rally in the capital. They needed bodies to listen to one of the president's Flag Day speeches. » (Danticat, The Dew Breaker, p. 163).

9. Danticat, The Dew Breaker, p. 164. Ceci rappelle l'Apocalypse de Saint-Jean 8, 6-13, La Bible de Jérusalem, Paris, Desclée De Brouwer, 1975, pp. 2129-30.

10. Danticat, The Dew Breaker, p. 165.

11. Danticat, The Dew Breaker, p. 161.

12. Danticat, The Dew Breaker, p. 161.

13. Danticat, The Dew Breaker, pp. 184-185.

14. «He was told to release the president. The change of orders had come directly from the national palace. [...] It was supposed to be a quiet operation, his superior, Rosalie, a short, stout, bespectacled woman told him. » (Danticat, The Dew Breaker, p. 184).

Danticat, The Dew Breaker, p. 185.

15. Danticat, The Dew Breaker, pp. 185-186.

16. Danticat, The Dew Breaker, p. 186.

17. Danticat, The Dew Breaker, pp. 186-7 ; nous soulignons.

18. Danticat, The Dew Breaker, p. 157.

19. Danticat, The Dew Breaker, p. 158.

20. Danticat, The Dew Breaker, pp. 170-1.

21. Danticat, The Dew Breaker, p. 169.

22. " 'I know my God and I'm placing myself in His hands', the preacher said as he devoured his supper of four squares of pulpy bread and a steaming cup of ginger tea. The preacher was dressed in his best cream jacket and vest ensemble [...]. At his long mahogany table, which he'd designed and built himself for meals with church members, the preacher was surrounded by three of his deacons who were trying to convince him to cancel the evening service and stay home. [...] Rising from his chair, he picked up his Bible, a leather-bound monogrammed volume, and thumped it against his palm as if to pound away his last shreds of doubt about going into the night. (Danticat, The Dew Breaker, pp. 169-71).

23. Danticat, The Dew Breaker, p. 170.

24. "But someone needed to stir the flock out of their stupor, the comfort that religion allowed them, that it was okay to have wretched lives here on earth so long as Heaven was glowing ahead. Maybe his death would do just that, move his people to revolt, to demand justice for themselves while requesting it for him. Or may be his death would have no relevance at all. He would simply join a long list of martyrs and his name would vanish from his countrymen's lips as soon as his body was placed into the ground. » (Danticat, The Dew Breaker, pp. 193-4).

25. Emmanuel Constant a été à l'origine de la création du FRAPH (Front Révolutionnaire Armé pour le Progrès d'Haïti), sur le modèle des Tontons Macoutes de l'ère Duvalier, pendant le gouvernement brutal de Raoul Cédras (1991-1994).

26. Danticat, The Dew Breaker, p. 66 et p. 70.

27. Danticat, The Dew Breaker, p. 62 et p. 67.

28. Danticat, The Dew Breaker, p. 68 et p. 72.

29. Danticat, The Dew Breaker, pp. 12-3.

30. Voir le vocabulaire violent: " holds on to it so tightly ", " his fingers crushing the bone ", " almost splitting it », « still throbbing ", " pain », " the ache », etc. (Danticat, The Dew Breaker, p. 15).

31. Danticat, The Dew Breaker, pp. 3-4.

32. Danticat, The Dew Breaker, pp. 16-7.

33. Danticat, The Dew Breaker, p. 18. 
34. " As my mother is speaking, this feeling comes over that I sometimes have when I'm carving, this sensation that my hands don't belong to me at all, that something else [...] is moving my fingers [...], an invisible puppetmaster over whom I have no control. I feel as though it's this same puppetmaster that now forces me to lower the hone and hang up, in midconversation, on my mother. " (Danticat, The Dew Breaker, p. 20).

35. Nadine elle-même, qui travaille - ironiquement - dans un service de laryngectomie, ne peut verbaliser son propre trauma, notamment par téléphone.

36. Danticat, The Dew Breaker, p. 88 et p. 102.

37. Anna Maria Karczewska, « Against Dechoukaj: The Trauma of Haiti in Edwidge Danticat's The Dew Breaker", Crossroads, A Journal of English Studies, 2015, Issue 11, http:// www.crossroads.uwb.edu.pl/dechoukaj-trauma-edwidge-danticats-dew-breaker/ , consulté en mars 2018.

38. Robert Birnbaum, «Edwidge Danticat », (from Morning News, April 20, 2004), reproduit dans Lavon Montgomery, Maxine (Ed.), Conversations with Edwidge Danticat, Jackson, University Press of Mississipi, 2017; également publié en ligne : https://themorningnews.org/article/birnbaum-v.edwidge-danticat .

39. Kali Tal, Worlds of Hurt. Reading the Literatures of Trauma. Cambridge, Cambridge University Press, 1996. Cité par A.M. Karczewska.

40. On y retrouve par exemple le traumatisme qu'a représenté l'arrestation de son oncle pasteur et la violence qui s'est déroulée dans l'église même de ce dernier, violence qui niait la sacralité même des lieux, puisqu'une église aurait dû être un sanctuaire. Voir l'interview accordée par E. Danticat à E. Ethelbert Miller, «An interview with ED », publiée dans Foreign Policy in Focus, October 16, 2007, et reproduite dans Maxine Lavon Montgomery (Ed.), pp. 34-6.

41. Le déchoukaj désigne les représailles à l'encontre des anciens Tontons Macoutes et responsables de la dictature.

Épisode rapporté par R. Birnbaum, p. 19.

42. Gallagher, « Concealment, Displacement \& Disconnection », p. 157.

43. Voir Florence Labaune-Demeule, « Le briseur de rosée : briseur de vies, briseur de voies/voix. Présences spectrales dans The Dew Breaker d'Edwidge Danticat ", in Béatrice Bijon, et Yves Clavaron, La production de l'étrangeté dans les littératures postcoloniales, Paris, Honoré Champion, 2009, p. 153.

44. Gallagher, "Concealment, Displacement \& Disconnection”, p. 18.

45. Gallagher, "Concealment, Displacement \& Disconnection", pp. 158-9.

46. E. Danticat, entretien avec Opal Palmer Adisa, 2009, «Up Close and Personal: Edwidge Danticat on Haitian Identity and the Writer's Life », in Maxine Lavon Montgomery (Ed.).

47. Édouard Glissant, Discours antillais, Paris, Gallimard, 1997, pp. 27-28.

48. Édouard Glissant, Traité du Tout-Monde, Paris, Gallimard, 1997, p. 121.

\section{RÉSUMÉS}

Dans son roman The Dew Breaker (2004), Edwidge Danticat décrit la dictature Duvalier ayant sévi à Haiti entre 1957 et 1986. A travers une étude du discours, cet article se propose d'explorer les difficultés rencontrées lorsqu'il s'agit de dénoncer la censure, de résister au pouvoir, ou simplement de dire l'horreur d'une réalité insupportable et du trauma dans une autocensure 
inconsciente. Dans un premier temps, on y questionne le lien entre la voix et la censure, ce qui conduit, dans un second temps à montrer comment le trauma lié à la censure et à la violence dictatoriales rend la parole impossible, condamnant victimes et bourreau à une autocensure et à des formes indirectes de discours. Enfin, cet article s'interroge sur la narration comme discours de révélation reposant paradoxalement sur des stratégies de discours implicite.

In her novel entitled The Dew Breaker (2004), Edwidge Danticat describes the Duvalier dictatorship that prevailed in Haiti between 1957 and 1986. Through a study of discourses, this essay aims at exploring how difficult it is to denounce censorship, to enter into dissidence, or simply to express the horror of such an unbearable reality or the trauma which entails unconscious selfcensorship. This article first questions the links between voice and censorship in order to show how the trauma generated by censorship and dictatorial violence makes it difficult to voice horror, compelling victims and torturers to censor themselves or to use deviant forms of discourse. Finally this essay considers the novel as a discourse of revelation which paradoxically relies on implicit discourse strategies.

\section{AUTEUR}

\section{FLORENCE LABAUNE-DEMEULE}

Florence Labaune-Demeule est Agrégée et Professeur des Universités en anglais et en littératures postcoloniales à l'Université Jean Moulin Lyon 3, Lyon, France. Plus particulièrement spécialisée dans les littératures postcoloniales caribéennes et indiennes, elle a publié de nombreux articles sur des auteurs comme V.S. Naipaul, Derek Walcott, Jean Rhys, Fred D'Aguiar, Edwidge Danticat, Jamaica Kincaid, ou Anita Desai, Anita Nair, Arundhati Roy, etc. Elle a également publié des ouvrages, dont une monographie consacrée à The Enigma of Arrival de V.S. Naipaul (V.S. Naipaul. L'Énigme de l'arrivée. L'éducation d'un point de vue, 2007), et plusieurs recueils d'articles.

Florence Labaune-Demeule is Professor in English and Postcolonial Literatures at Jean Moulin Lyon 3 University, Lyon, France. Her research interests focus on Anglophone Caribbean and Indian literatures. She has written extensively on writers of Caribbean or Indian descent like V.S. Naipaul, Derek Walcott, Jean Rhys, Fred D'Aguiar, Edwidge Danticat, Jamaica Kincaid, Anita Desai, Anita Nair, Arundhati Roy, etc. She has also published a few books, among which a monograph about V.S. Naipaul's The Enigma of Arrival (V.S. Naipaul. L'Énigme de l'arrivée. L'éducation d'un point de vue, 2007) and a few collections of essays. 